\title{
PREDICTING HOUSING SALES IN TURKEY USING ARIMA, LSTM AND HYBRID MODELS
}

\author{
Ayşe SOY TEMÜR (iD) ${ }^{*}$, Melek AKGÜN² , Günay TEMÜR (ID) \\ ${ }^{1}$ Institute of Social Sciences, Sakarya University, Sakarya, Turkey \\ ${ }^{2}$ Faculty of Business Administration, Sakarya University, Sakarya, Turkey \\ ${ }^{3}$ Computer Engineering, Düzce University, Düzce, Turkey
}

Received 01 October 2018; accepted 18 April 2019

\begin{abstract}
Having forecast of real estate sales done correctly is very important for balancing supply and demand in the housing market. However, it is very difficult for housing companies or real estate professionals to determine how many houses they will sell next year. Although this does not mean that a prediction plan cannot be created, the studies conducted both in Turkey and different countries about the housing sector are focused more on estimating housing prices. Especially the developing technological advances allow making estimations in many areas. That is why the purpose of this study is both to provide guiding information to the companies in the sector and to contribute to the literature. In this study, a 124-month data set belonging to the 2008 (1)-2018 (4) period has been taken into account for total housing sales in Turkey. In order to estimate the time series of sales, ARIMA (Auto Regressive Integrated Moving Average as linear model), LSTM (Long Short-Term Memory as nonlinear model) has been used. As to increase the estimation, a HYBRID (LSTM and ARIMA) model created has been used in the application. When MAPE (Mean Absolute Percentage Error) and MSE (Mean Squared Error) values obtained from each of these methods were compared, the best performance with the lowest error rate proved to be the HYBRID model, and the fact that all the application models have very close results shows the success of predictability. This is an indication that our study will contribute significantly to the literature.
\end{abstract}

Keywords: house sales forecast, hybrid model, recurrent neural network, ARIMA, LSTM network, data estimation methodology, time series analysis, housing sales in Turkey.

JEL Classification: C45, C53, C89, D1.

\section{Introduction}

In order for supply and demand in the housing market to be balanced, it is very important that housing companies and real estate professionals determine how many houses will be sold in the next year. The need for housing (shelter) is one of the basic needs along with physi-

${ }^{\star}$ Corresponding author. E-mail: aysesoy@duzce.edu.tr 
ological needs such as breathing, drinking water and eating. That's why housing is not only an economic value but also an asset with socio-psychological characteristics.

Due to the housing sales, for many modern economies the construction sector is the catalyst of the economy and is seen among economic growth figures. For example, capital investments for housing in the US are higher than those for new ventures (Greenwood \& Hercowitz, 1991). The housing market, which is an important scale for the national economy, has a positive effect on the currency of the country. New housing supply creates an economic wave effect such as homeowners buying goods like household appliances or furniture for their own homes, builders buying raw materials to meet the demands and leasing more employees. The high level of housing supply shows that the construction industry is healthy and that consumers have capital to make large investments. In addition, the housing sector continues to be an "investment instrument" as well as "housing in certain conditions." The most important external resource that makes housing investment easier in a certain term are bank loans and their interest rates. The interest variable is critical for sales and all the parameters that depend on it.

The financial crisis that started with the mortgage crisis stemming from the housing and real estate market in the US in August 2007 became a global economic crisis starting from September 2008. Along with the US economy, the global economic crisis of 2008 negatively affected the economy of many countries as well, especially developing countries, in a chain reaction. This is an important indicator of the fact that the housing sector can adversely affect individuals as well as many sectors, especially banks. The examination of the housing market has become even more important after the explosion of the real estate bubble, which triggered the economic crisis in the rest of the world, especially in the US.

The recovery of the housing market, which directly or indirectly affects many sectors in the economy, may be an indication of the recovery of the country's economy as well (Wu \& Brynjolfsson, 2015). Economists, politicians, bankers and investors generally use historical data published by the competent authorities of the state to assess the current situation of the housing market and make predictions for the future. However, the publication of these data takes time and this causes a delay in the evaluation of the current economic conditions. These delays have a negative impact on firms operating in sectors such as home appliances and furniture which are affected by the housing sector, as well as firms in the construction sector.

Studies related to the housing sector in Turkey are usually conducted for the estimation of housing prices, and no study has not been encountered on the subject of prediction of housing sales that vary depending on the prices. In a data search regarding international studies, it is seen that $\mathrm{Wu}$ and Brynjolfsson (2015) conducted a research on the housing market to show how data on internet queries can be used to make reliable estimates of both quantities and prices. In that study, data about the behaviors of individuals who wanted to buy housing using the Google search engine were analyzed, housing sales and housing prices were estimated. In addition, it was discovered in the study that housing sales and house price index were positively related. Since the effects of the search volume on the housing price index are uncertain, it is mentioned that the index estimate may be more difficult than the sales forecast. In addition to that, the total volume of the houses sold proved to be related to the 
purchase demands of home appliances (Wu \& Brynjolfsson, 2015). This is an indication that accurate estimation of housing sales will also provide useful information for many sectors.

In this study, ARIMA has been preferred as a linear model because of its use as one of the methods frequently used in social sciences for the estimation of housing sales. Plus, LSTM, which has started to be widely used in recent years, has been preferred as the non-linear model because it covers for the shortcomings of RNN. The application of the same data to more models rather than one can increase the accuracy of the estimation. Therefore, a hybrid model has been created to overcome the limitations of LSTM and ARIMA models. 124 units of data published by Turkey Statistical Institute (TUIK) which includes monthly housing sales in Turkey between 01.2008 and 04.2018 were used in the study as data set.

The main goal of this study is to provide reliable and accurate estimation studies in the housing sector by using methods with a scientific basis and to ensure that housing sales are estimated as close as possible to the real value. This will help to obtain information to support the decision-making process of shareholders in the sector. Therefore, analyses were conducted firstly with ARIMA model, secondly with LSTM model and lastly with a hybrid model which was created by combining the advantages of the two aforementioned models. Afterwards, the results obtained from each of the three methods were evaluated one by one and compared with each other. These methods do not pose any restrictions and it is possible to make future estimations by using different methods related to house sales. The estimation results obtained will contribute to the future estimations for many industries related to housing sector and help us to be more informed about the progress of the country's economy. In addition, this study aims to contribute to the literature for the benefit of similar studies to be carried out.

The structure of the paper follows: The first section is the literature section and it includes certain examples of projects which have used ARIMA, LSTM and some Hybrid models. The second section describes the methodology used to create predictions. In the third section, information about the data set and application procedures are explained. Section four consists of the output results of the study and information about the general evaluation of the findings can be found in section five.

\section{Literature review}

In the past decades, much of the social science research focused on refining increasingly complex mathematical models to predict social and economic trends. There are many methods available in the time series analysis, all with their own advantages and disadvantages, and these methods can be explained as techniques of creating predictions and policies about future values using past data.

ARIMA models have been applied in the literature to predict future values of various time series data such as electricity prices, sugar prices, house prices, stock quotes, wind speeds, water quality and global temperature values. ARIMA models can help understanding the dynamics of a given application. For example, Ediger and Akar predicted the primary energy demand for the period 2005-2020 using the data of 1950-2004 with ARIMA model (Ediger \& Akar, 2007). Albayrak predicted the primary energy production and demand for 
the period 2007-2015 using the data of 1923-2006 period with ARIMA model (Albayrak, 2010). Erdoğdu predicted the electricity consumption for the period 2005-2014 using the data of 1923-2004 period with ARIMA model (Erdoğdu, 2007).

On the other hand, LSTM networks are used mostly for deeper learning and they achieve greater success with large data sets. However, though limited in number, examples of LSTM being trained with few data sets are also available in the literature. For example, Namin and Namin tried an LSTM network model on the prediction of economic and financial time series in 2018. As a result of this trial, they again achieved success with a $13 \%-16 \%$ error margin (S. S. Namin \& A. S. Namın, 2018).

Some of the studies on hybrid models combining the advantages of two or more individual models in the literature can be summarized as follows.

Zhang (2003) conducted a study on the estimation of time series using ARIMA and neural network hybrid model. Wolf's sunspot data, Canadian lynx data and British pound/US dollar exchange rate data were used in the study. With the hybrid model he developed in this study, Zhang demonstrated that neither ARIMA nor ANN is suitable for all real time series, and that there are linear and nonlinear correlation structures between observations in these series and therefore a hybrid model should be used to estimate both linear and nonlinear components of a time series (Zhang, 2003).

Khashei (2008), in their work using ANN and fuzzy regression methods, have proposed a hybrid model that provides more accurate results with missing data sets. In the proposed model, the advantages of ANN and fuzzy regression have been combined to overcome the limitations in both ANN and fuzzy regression. To show the suitability and effectiveness of the method, it was used in the estimation of the price of gold (Gram/US\$) and exchange rate (US\$/Iran Rials). The results showed that the proposed model could be an effective way to improve estimation accuracy (Khashei, 2008). Aladağ, Eğrioğlu, and Kadılar (2009) proposed a new hybrid approach with the Elman recurrent neural networks (RNN) and seasonal ARIMA (SARIMA) models. In this proposed hybrid model, Canadian lynx data for the period 1821-1934 were used, which consisted of the annual number of lynx traps in the Mackenzie River area in Northwest Canada. Although the data used were very limited, the hybrid method gave the best estimation accuracy in the application results (Aladağ et al., 2009). Koutroumanidis, Ioannou, and Arabatzis (2009), in their studies to examine the role of forests in firewood production in Greece, predicted the future situation of the sale prices of wood produced by the Greek state forest farms. They used ARIMA, ANN and HYBRID models for estimation and obtained the best estimation results using the ARIMA-ANN HYBRID model (Koutroumanidis et al., 2009).

Koutroumanidis, Ioannou, and Zafeiriou (2011) aimed to establish confidence intervals for predicted values of a time series in their studies for predicting stock market prices with hybrid method. Daily closing prices of the shares of Alpha Bank from 28/01/2004 to $30 / 11 / 2005$ were used as samples of the study. For the estimation, ANN was applied to the raw data and then the market prices were estimated using the Bootstrap method. Estimation accuracy was measured by using different criteria and satisfactory results were obtained (Koutroumanidis et al., 2011). Ioannou, Birbilis, and Lefakis (2011) presented a method for estimating the likelihood of the Ring Shake appearance in planted chestnut trees. In his 
research, it was claimed that the ring shake phenomenon in Castanea sativa caused a reduction in the production of chestnut wood in Europe and although it was not certain, it was claimed that age and annual growth were the most important factors in the occurrence of this defect. They used the ANN method to estimate age and annual growth in their studies (Ioannou et al., 2011). He and Deng (2012) developed a hybrid model using ARIMA and ANN to estimate air pollutant factors. Firstly, ARIMA and ANN was used to estimate the time series and then a re-estimation was done with the hybrid model that was developed. When the results were compared, it became clear that the hybrid model performed better (He \& Deng, 2012).

Papagera, Ioannou, Zaimes, Iakovoglou, and Simeonidou (2014) MIKE conducted a research on water balance estimation by using SHE and ANN models. A 4-year data set for the 2008-2012 years of Lake Koronia in the northern part of Greece was used for the study (Papagera et al., 2014). Babu and Reddy (2014) investigated the nature of volatility by using experimental and simulated data sets such as sunspot, electricity price and stock market data. They first used moving average filter, and later ARIMA and ANN models were applied. A hybrid model was proposed along with ARIMA and ANN models used in the application and some existing HYBRID ARIMA-ANN models. The results from the data sets show that the hybrid model has a higher estimation accuracy for both single-step and multi-step predictions (Babu \& Reddy, 2014). Hocaoğlu, K. Kaysal, and Kaysal, A. (2015) used the hybrid model they created using ANN and regression methods, for load estimation in the energy sector. When the error results are compared, it is concluded that the hybrid system has the least amount of errors (Hocaoğlu et al., 2015).

Pablo et al. (2016) proposed a hybrid approach to the reconstruction of the time series with the creation of ANN and Monte Carlo Simulation. They tried to estimate the daily milk sales of a dairy company using these models. The results show that the proposed method can reconstruct the past and predict the future from the known time series segment (Pablo, et al., 2016). Sugiartawan, Pulungan, and Sari (2017) used a hybrid model that they created with wavelet transform and LSTM in order to predict the number of tourists coming to Indonesia over a monthly period. The prediction results of the proposed hybrid model were compared with other RNN algorithms, namely ELMAN RNN and Jordan RNN and the hybrid of Elman's wavelet and the hybrid of Jordan's wavelet. It was concluded that the hybrid model generated from wavelet transform and LSTM gives a better training duration than the original LSTM, Elman and Jordan RNNs and predicts the number of arriving tourists more accurately than other hybrid methods (Sugiartawan et al., 2017). Lin, Guo, and Aberer (2017) inspired by the recent successes of artificial neural networks, proposed TreNet, a completely new hybrid neural network, to predict the trend of time series. They used three different data sets in their study: electricity consumption, chemical sensor records subject to dynamic gas mixtures at variable concentrations, and daily stock trading information on Yahoo Finance and the New York Stock Exchange. At the end of the study, they stated that TreNet could be used to predict trend evolution in time series (Lin et al., 2017).

To predict second-hand house prices in Beijing, Yu, Jiao, Xin, Y. Wang, and K. Wang (2018) used the Convolution Neural Network (CNN) and Long Short-Term Memory (LSTM) models based on deep learning and the Auto-Regressive and Moving Average (ARMA) mod- 
el of time series. The results obtained from CNN, LSTM and ARMA models were compared. They applied a logical regression model to compare the three models used. They concluded that the prediction accuracy of the LSTM, which takes the time series into account, is better than other methods (Yu et al., 2018). ARIMA-LSTM hybrid model has been used to estimate the stock correlation coefficient by Choi (2018). The performance of this hybrid model has been determined superior to other conventional financial models by the tests performed (Choi, 2018). Sagheer and Kotb (2019), using DLSTM model have estimated the production data of two real oil fields. When they compared the performance of the proposed approach with the different models, they concluded that the DLSTM model performed better (Sagheer \& Kotb, 2019). Xu et al. (2019) have used a linear regression and deep learning hybrid model for time series estimation. They have concluded that the hybrid model has a higher estimation accuracy when compared to other models. Therefore, they have stated that the proposed hybrid model can be a useful tool for time series estimation (Xu et al., 2019).

\section{Research methodology}

This section of the study will discuss the ARIMA and LSTM models used for prediction and the basic principles and modeling processes of the hybrid model obtained by combining these models in a rational way.

\subsection{ARIMA}

The ARIMA method consists of three main processes: diagnostic check, identification and prediction. In the first step called diagnostic check, stationarity control is performed on the given time series data. Stationary time series is a time series in which statistical properties such as mean, variance and covariance are according to time. Stationarity is essential when creating the ARIMA model which makes the prediction very practical and useful. In order to make a non-stationary time series stationary, differencing $(d)$ at an appropriate degree is performed and the stability is tested again. This process continues until a stationary series is obtained. $(d)$ is a positive integer and is responsible for the differencing degree. If the differencing operation is performed $(d)$ times, the integration parameter of the ARIMA model is set to $(d)$. Then, identification is performed on the stationary data obtained. In this process, the parameters of the autoregressive (AR) and moving average (MA) operations shown in equation (1) are determined as $(p)$ and $(q)$, respectively. An ARIMA model is defined as $\operatorname{ARIMA}(p, d, q)$ (Newbold, 1983).

$-p$ : degree of autoregressive model (AR)

$-d$ : differencing degree

- $q$ : degree of moving average model (MA)

$$
y_{t}=\alpha_{1} w_{t-1}+\alpha_{2} w_{t-2}+\ldots+\alpha_{p} w_{t-p}+\varepsilon_{t}-\theta_{1} \varepsilon_{t-1}-\theta_{2} \varepsilon_{t-2}-\ldots-\theta_{q} \varepsilon_{t-q} .
$$

For the $t$ time here, $y_{t}$ denotes the linearized real data while $\varepsilon_{t}$ denotes the moving average error. As shown in the formula, a linear relationship has been established between actual data $y_{t}$ to be predicted, the observed $(p)$ data $\left(y_{t-1}, y_{t-2}, \ldots, y_{t-p}\right)$, and $(q)$ error data $\left(\varepsilon_{t}\right.$, $\left.\varepsilon_{t-1}, \ldots, \varepsilon_{t-q}\right)$. 


\subsection{LSTM}

LSTMs are a special type of RNN designed to learn long-term dependencies. They were first developed by Hochreiter and Schmidhuber (1997). It has a complex structure called the LSTM unit in the hidden layer it contains. A simple representation of this structure is given in Figure 1. Since they work very well on a wide variety of problems, they are widely used today.

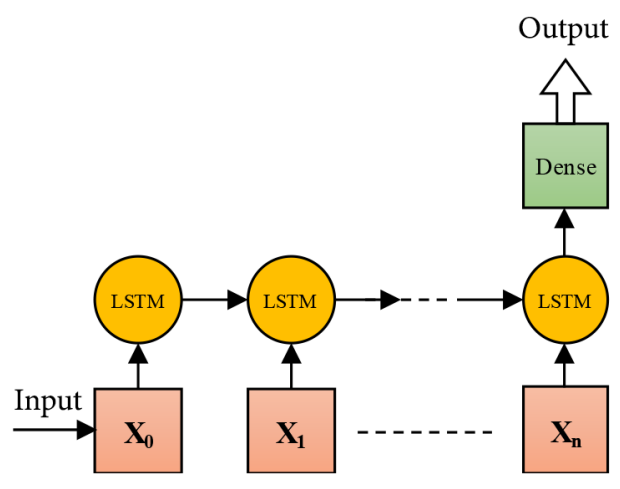

Figure 1. LSTM architecture (Atienza, Posted on 2017)

To roughly describe, in an LSTM structure, there is also a memory along with the RNN cell. Thanks to this memory, information from the previous time can be retrieved and transmitted to the next one. The model decides which information to take with training. Remembering information for a long time is in practice the default behavior of these networks and not something they try to learn. An LSTM unit is shown in Figure 2.

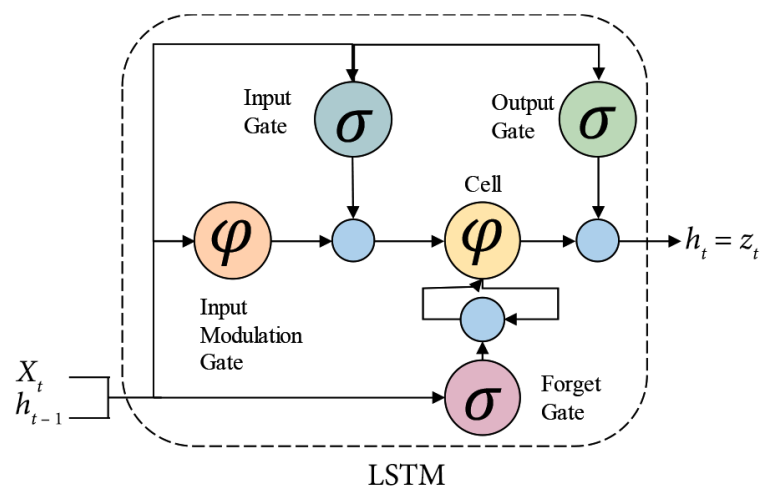

Figure 2. LSTM structure (Kang, 2018)

Here $X_{t}$ represents the input data at the $t$ time step and the output of the previous unit. $h_{t}$ is hidden units output while $h_{t-1}$ is their previous output. For the LSTM unit, input gate $i_{t}^{j}$ (2) forget gate $f_{t}^{j}(3)$ and output gate $\sigma_{t}^{j}(4)$ equations may be used to calculate.

$$
i_{t}^{j}=\sigma\left(W_{x i} x_{t}+W_{h i} h_{t-1}+b_{i}\right)^{j}
$$




$$
\begin{gathered}
f_{t}^{j}=\sigma\left(W_{x f} x_{t}+W_{h f} h_{t-1}+b_{f}\right)^{j} ; \\
\sigma_{t}^{j}=\sigma\left(W_{x \sigma} x_{t}+W_{h \sigma} h_{t-1}+b_{\sigma}\right)^{j},
\end{gathered}
$$

Here; $\sigma$ is sigmoid function, $w$ terms are weight matrices and $b$ terms are voltage vectors. Unlike the traditional epoch unit, each $j$. LSTM unit preserves its memory at $t$ time with $\left(c_{t}^{j}\right)$. Here, the equation whose memory cell is given is updated via equation (5).

$$
c_{t}^{j}=f_{t}^{j} c_{t-1}^{j}+i_{t}^{j} c_{t}^{\sim j} .
$$

The new memory content is updated with equation (6) and the output for the LSTM unit is calculated by equation (7).

$$
\begin{gathered}
f_{t}^{j}=\tanh \left(W_{x c} x_{t}+W_{h c} h_{t-1}+b_{c}\right)^{j} ; \\
h_{t}^{j}=\sigma_{t}^{j} \tanh \left(c_{t}^{j}\right) .
\end{gathered}
$$

As in other ANNs, training is carried out on LSTM networks by epoch. An epoch specifies the total number of iterations of a given set of data used for training purposes in the calculation of network weight values $(w)$. An epoch refers to the fact that an entire data set has passed forward and then back on the network.

Updating weights to optimize models of deep learning algorithm, and thus transmitting the entire data set over a single network many times to obtain a better and more accurate prediction model is sensible. However, it is not clear how many epoch numbers will be needed to achieve optimal weights and to train a model with the same data set. Different sets of data exhibit different behaviors, so a different number of epochs may be needed to best train networks.

\subsection{Hybrid method}

Many time series models include linear relationships as well as nonlinear relationships. While the ARIMA models are good at modeling the linear relationship in the time series, they are insufficient at modeling nonlinear relationships. The LSTM models can model both linear and nonlinear relationships but cannot provide the same results for each data set. For this reason, in order to reach the best prediction results, hybrid models based on the principle of separate modeling of linear and nonlinear components of time series are employed. These models have achieved great successes in forecasting time series analysis; use multiple learning algorithms to achieve better estimation performance than from constructive learning algorithms (Opitz \& Maclin, 1999). These models are supervised learning algorithms because they are available for training and for predictive purposes. The purpose of these models is to increase the diversity of models and to achieve better results (Adeva, Beresi, \& Calvo, 2005; Oliveira \& Torgo, 2014).

The results obtained by using the hybrid models and the results obtained from the individual use of models, even though they are unrelated to each other, it was observed that they can reduce the general variance or error (Khashei, 2008). For this reason, hybrid models are recognized as the most successful models of forecasting tasks. 
Different studies used many hybrid models consisting of linear models and nonlinear models for prediction purposes. In this study, created to make predictions about the future by using historical data from a time series The ARIMA-LSTM hybrid model given in Figure 3 was used.

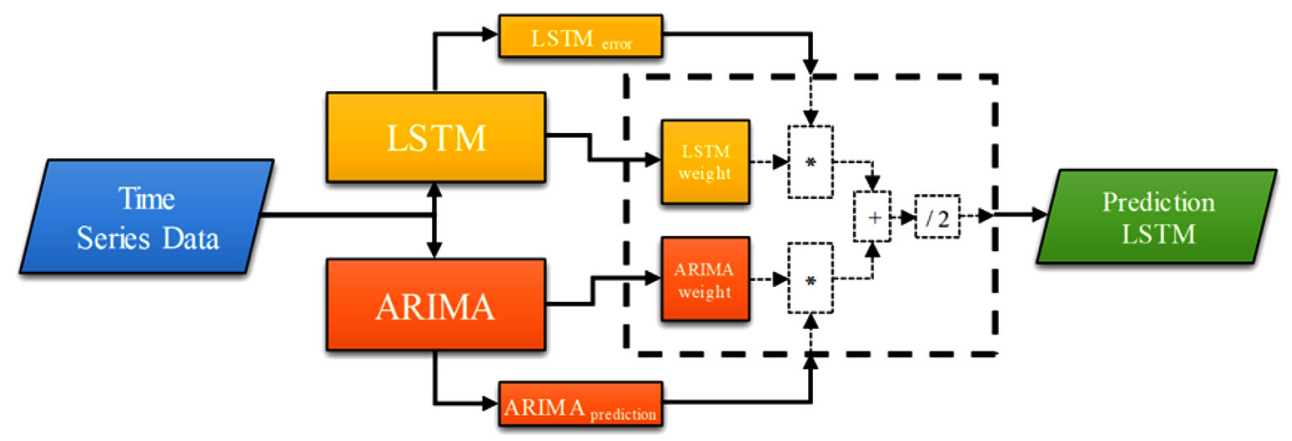

Figure 3. Hybrid model diagram

The time series prediction formula of the generated model can generally be expressed as the sum of linear and nonlinear components, as shown in equation (8) (Zhang, 2003).

$$
y_{t}=L_{t}+N_{t} .
$$

$L_{t}$ shows the linear component of the time series whereas $N_{t}$ shows the non-linear component. In the hybrid model, the linear component of the time series is predicted first using the $L_{t}$ ARIMA model then the $N_{t}$ LSTM model. Then error values of both models are calculated. The formula for this calculation is given in Equations (9) and (10).

$$
\begin{gathered}
l s t m_{\text {error }}=l s t m_{-} \text {mean }[\text { error }] \\
\text { arima }_{\text {error }}=\text { arima_mean }[\text { error }] .
\end{gathered}
$$

The weights of the models were calculated by using the obtained error values in Equations (11) and (12).

$$
\begin{gathered}
l s t m_{\text {weight }}=\left(1-\left(\frac{\text { stm }_{\text {error }}}{\text { lstm }_{\text {error }}+\text { arima }_{\text {error }}}\right)\right) * 2 ; \\
\text { arima }_{\text {weight }}=2-\text { lstm }_{\text {weight }} .
\end{gathered}
$$

The weight values of the models and finally each prediction value of the hybrid model are obtained with the given equation (13).

$$
\text { hibrit }_{\text {predict }}[i]=\left(\operatorname{lstm}_{\text {weight }}[i] \times l \operatorname{stm}_{\text {error }}[i]+\text { arima }_{\text {weight }} \times \text { arima }_{\text {predict }}[i]\right) / 2 .
$$




\subsection{Success criteria of models}

No matter which of the prediction methods is used, they do not produce $100 \%$ accurate value. In any case, if the future is known $100 \%$, it would not be a prediction. Therefore, each prediction has a certain error rate. Data-compatible model and high prediction success are two of the most widely accepted criteria in the process of selecting one from among various prediction models. There are several criteria that compare the predictive successes of models. The most important of these criteria is the accuracy of prediction. The accuracy of the prediction method is measured by analyzing the predicted errors (Sar1, 2016).

In order to measure the model predictive success of the 3 methods in this study, Mean Squared Error (MSE), Root Mean Squared Error (RMSE), Mean Absolute Percentage Error (MAPE), and Mean Absolute Error (MAE) criteria have been used. The formulas used to calculate these criteria are given in Table 1 (Sallehuddin, Shamsuddin, Hashim, \& Abraham, 2007).

Table 1. Success criterion (corelogram) formulas

\begin{tabular}{|l|c|}
\hline Name & Formulas \\
\hline Mean Square Error & $M S E=\frac{1}{n} \sum_{t=1}^{n}\left(y_{t}-\hat{y}_{t}\right)^{2}$ \\
\hline Root Mean Square Error & $\sqrt{\frac{1}{n} \sum_{t=1}^{n}\left(y_{t}-\hat{y}_{t}\right)^{2}}$ \\
\hline Mean Absolute Error & $\left(\sum_{t=1}^{n}\left|\frac{y_{t}-\hat{y}_{t}}{n}\right|\right)$ \\
\hline Mean Absolute Percentage Error & $\left(\sum_{t=1}^{n}\left|\frac{y_{t}-\hat{y}_{t}}{y_{t}}\right|\right) \frac{100}{n}$ \\
\hline
\end{tabular}

Here $y_{t}$ denotes the real value, $\hat{y}_{t}$ predicted value, $n$ the number of the predicted period. The model that has the lowest criteria values obtained with the above formulas should be selected as the most suitable model.

\section{Data and research findings}

In this part, the information about the data used in this study and the findings obtained from the results of the application are given.

\subsection{Data}

Within the scope of the study, the number of house sales for the period of 2008 (1)-2018 (4) in Turkey was arranged as x1000 and a total of 124 months data set was used. The dataset used was obtained from the website of TUIK (Turkish Statistical Institute), which is an 
open platform. The main reason for considering the data start year as 2008 is because the housing sales statistics began being published monthly on the TUIK website in 2008. 2018 includes the period data published until the implementation period. The related data set has been represented in a series to be used in the neural network model. The graph of the data represented by years is given in Figure 4.

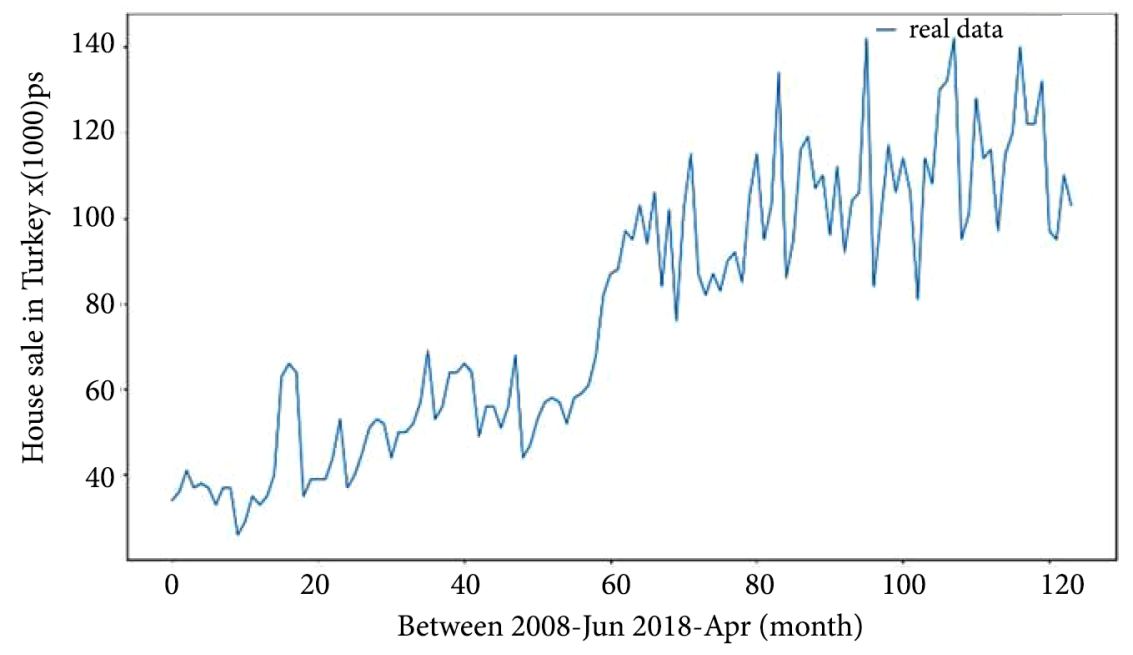

Figure 4. Monthly house sales data graphic

In the case of estimation applications, a data set is treated as Training and Test. The most important problem encountered in estimation applications is the amount of training and test data. Artificial neural networks should be trained using as much data as possible. The data not used in the training set is used in the test set. The output obtained by feeding the test data to the network is compared with the actual output values. The main objective is to check whether the power to represent the obtained sample is sufficient.

In the literature, $70 \%$ training, $30 \%$ test or $80 \%$ training and $20 \%$ test split of the data set is generally accepted in determining the training and test data. In this study, $70 \%$ of this data set was used for training and 30\% for test.

The data have been applied to the ARIMA, one of the linear methods commonly used in time series predictions, and the LSTM network, one of the nonlinear methods recently used for deep learning algorithms. In addition, hybrid methods generally accepted in the literature have been examined and a hybrid method based on ARIMA and LSTM has been tested for this study. All the methods have been coded in Python 3.6 programming language and open source libraries have been employed.

\subsection{ARIMA model}

7 different ARIMA (p,d,q) models were determined for the estimation of sales data realized by ARIMA estimation method and the corelogram analyses of these models were performed. The results of the success criteria and model error comparisons of the estimation studies are given in Table 2 and the significance tests have been performed both on this table and the 
output graphics formed by the models. According to the model results in the table, a model with a lower error rate is a more valuable model for prediction. Accordingly, ARIMA $(0,1,2)$ and ARIMA $(1,1,1)$ models with the lowest MAPE values were found to perform the most successful predictions among the models.

Table 2. ARIMA models correlogram results

\begin{tabular}{|c|c|c|c|c|}
\hline \multicolumn{5}{|c|}{ ARIMA Model Comparison } \\
\hline ARIMA & MAE & MSE & RMSE & MAPE \\
\hline $0,1,1$ & 12.894 & 252.509 & 16.808 & 0.122 \\
\hline $0,1,2$ & 12.801 & 280.400 & 16.745 & 0.121 \\
\hline $1,1,0$ & 14.895 & 345.399 & 18.585 & 0.140 \\
\hline $1,1,1$ & 12.835 & 282.599 & 16.811 & 0.121 \\
\hline $2,1,0$ & 13.630 & 328.938 & 18.137 & 0.128 \\
\hline $2,1,1$ & 13.275 & 293.463 & 17.131 & 0.126 \\
\hline $2,2,1$ & 13.823 & 337.640 & 18.375 & 0.130 \\
\hline
\end{tabular}

The comparative graph of the 36-month real and predicted values obtained with ARIMA $(0,1,2)$ model, which is one of the best estimate with the lowest MAPE value, is given in Figure 5.

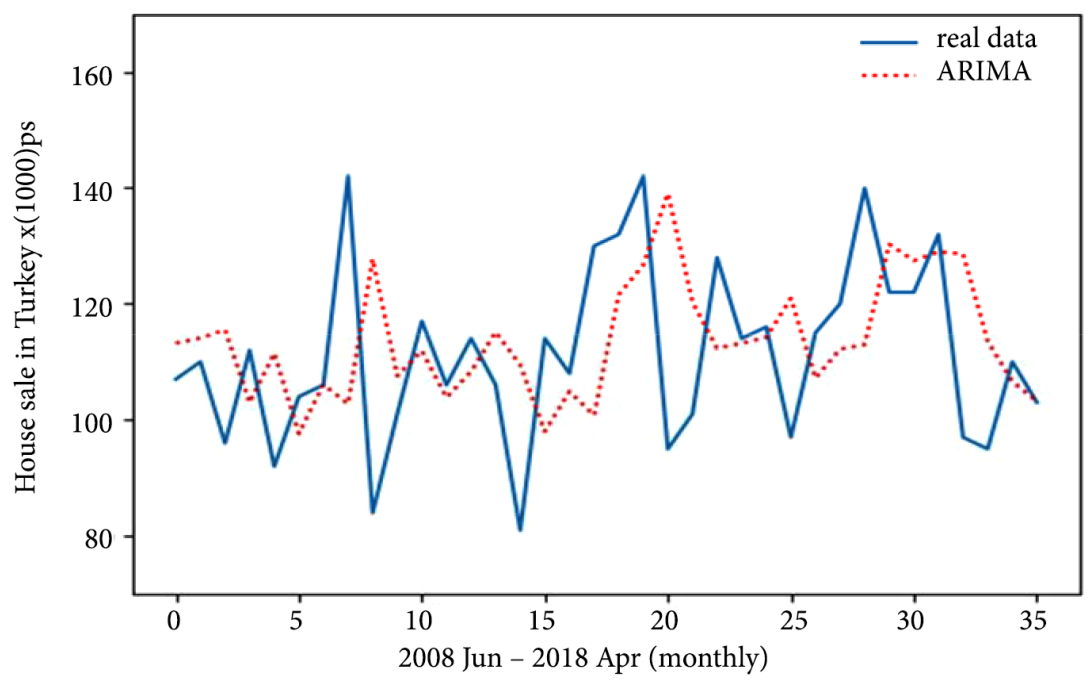

Figure 5. ARIMA $(0,1,2)$ model and ground truth

When Figure 5 is examined, it can be seen that the actual and predicted real estate sales values are overlapping with each other and the deviations between them do not show any excess. The success on the graph can be understood from the close values of the data as well as the similarity of directional breaks. The model used here is able to produce values close to the actual data with an error value of 0.121 MAPE. This shows the success of the applied model. 


\subsection{LSTM model}

In this part of the study, a Long Short-Term Memory-LSTM architecture based on the time series has been employed. Encoded in Python software, this part has been implemented with KERAS, a deep learning library used to develop the LSTM model. As is known, LSTM networks are a deep learning application and produce better results in situations where the number of data sets is too high. However, as noted under the heading of the literature, there are situations, albeit in a very small number, in which a simple LSTM architecture is trained with little data. The LSTM network takes the data in a sequential format and ensures that the ordering of sales values is taken into account in the training and classification steps. For the application, the LSTM network was run on the monthly house sales amounts of the 2008-2018 period. In this section, $70 \%$ of the data is again used for education and the remaining $30 \%$ for testing. During the training process, the resulting error values were observed with different epoch numbers. The error values that occur according to the Epoch numbers are given in Table 3.

According to the error values seen in Table 3, the result of the model trained with 1000 epoch has the lowest error rate. The graphical representation of the estimation results of this value is given in Figure 6.

Table 3. LSTM models correlogram results

\begin{tabular}{|c|c|c|c|c|}
\hline \multicolumn{5}{|c|}{ LSTM Model Comparison } \\
\hline Epoch & MAE & MSE & RMSE & MAPE \\
\hline 1000 & 17.953 & 473.346 & 21.757 & 0.150 \\
\hline 1500 & 19.322 & 571.857 & 23.914 & 0.161 \\
\hline 2000 & 19.937 & 584.803 & 24.183 & 0.167 \\
\hline 2500 & 19.096 & 530.578 & 23.034 & 0.162 \\
\hline 2750 & 19.549 & 553.538 & 23.527 & 0.166 \\
\hline
\end{tabular}

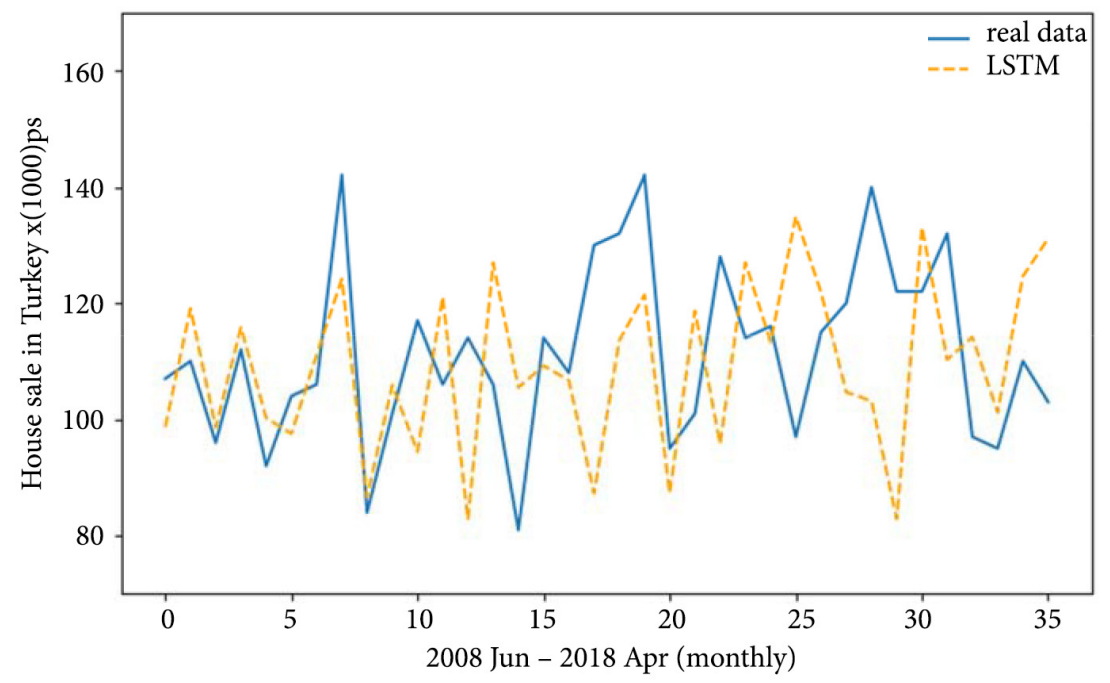

Figure 6. LSTM model (1000 epoch) and ground truth 
As the graph shows, the LSTM model produced good results with little data; but, with a MAPE value of 0.150 , which is a success criterion evaluation, it produced a higher error value than the ARIMA model. With this comparison, it can be said that ARIMA model is more successful than LSTM model.

\subsection{HYBRID model}

In this section, 1000-1500-2500 epoch valuable models in which the lowest MAPE values were obtained in the LSTM network and all models of ARIMA were applied to the HYBRID model designed in Figure 3 for re-estimation. The estimation results obtained with the HYBRID model are shown in detail in Table 4.

Table 4. HYBRID models correlogram results

\begin{tabular}{|c|c|c|c|c|c|}
\hline \multicolumn{6}{|c|}{ HYBRID Model Comparison } \\
\hline LSTM Epoch & $\operatorname{ARIMA}(\mathrm{p}, \mathrm{d}, \mathrm{q})$ & MAE & MSE & RMSE & MAPE \\
\hline \multirow{7}{*}{1000} & $0,1,1$ & 9.517 & 191.085 & 13.823 & 0.083 \\
\hline & $0,1,2$ & 9.243 & 183.905 & 13.561 & 0.081 \\
\hline & $1,1,0$ & 10.332 & 195.781 & 13.992 & 0.091 \\
\hline & $1,1,1$ & 9.182 & 182.589 & 13.513 & 0.080 \\
\hline & $2,1,0$ & 10.451 & 214.307 & 14.639 & 0.092 \\
\hline & $2,1,1$ & 9.406 & 188.232 & 13.720 & 0.082 \\
\hline & $2,2,1$ & 10.419 & 212.628 & 14.582 & 0.091 \\
\hline \multirow{7}{*}{1500} & $0,1,1$ & 8.805 & 185.136 & 13.606 & 0.075 \\
\hline & $0,1,2$ & 8.535 & 177.111 & 13.308 & 0.073 \\
\hline & $1,1,0$ & 9.662 & 190.100 & 13.788 & 0.083 \\
\hline & $1,1,1$ & 8.847 & 175.611 & 13.252 & 0.072 \\
\hline & $2,1,0$ & 9.679 & 205.799 & 14.346 & 0.083 \\
\hline & $2,1,1$ & 8.597 & 179.003 & 13.379 & 0.073 \\
\hline & $2,2,1$ & 9.623 & 203.491 & 14.265 & 0.082 \\
\hline \multirow{7}{*}{2500} & $0,1,1$ & 9.023 & 184.935 & 13.599 & 0.079 \\
\hline & $0,1,2$ & 8.640 & 173.458 & 13.170 & 0.076 \\
\hline & $1,1,0$ & 9.526 & 174.392 & 13.206 & 0.084 \\
\hline & $1,1,1$ & 8.576 & 171.160 & 13.083 & 0.075 \\
\hline & $2,1,0$ & 9.810 & 201.630 & 14.200 & 0.086 \\
\hline & $2,1,1$ & 8.759 & 174.965 & 13.227 & 0.077 \\
\hline & $2,2,1$ & 9.763 & 199.645 & 14.130 & 0.086 \\
\hline
\end{tabular}

Figure 7 shows the output of the prediction results of $\operatorname{LSTM}(1500$ epoch)-ARIMA $(1,1,1)$ model, recognized as the best prediction with the HYBRID Model, and the actual values. 


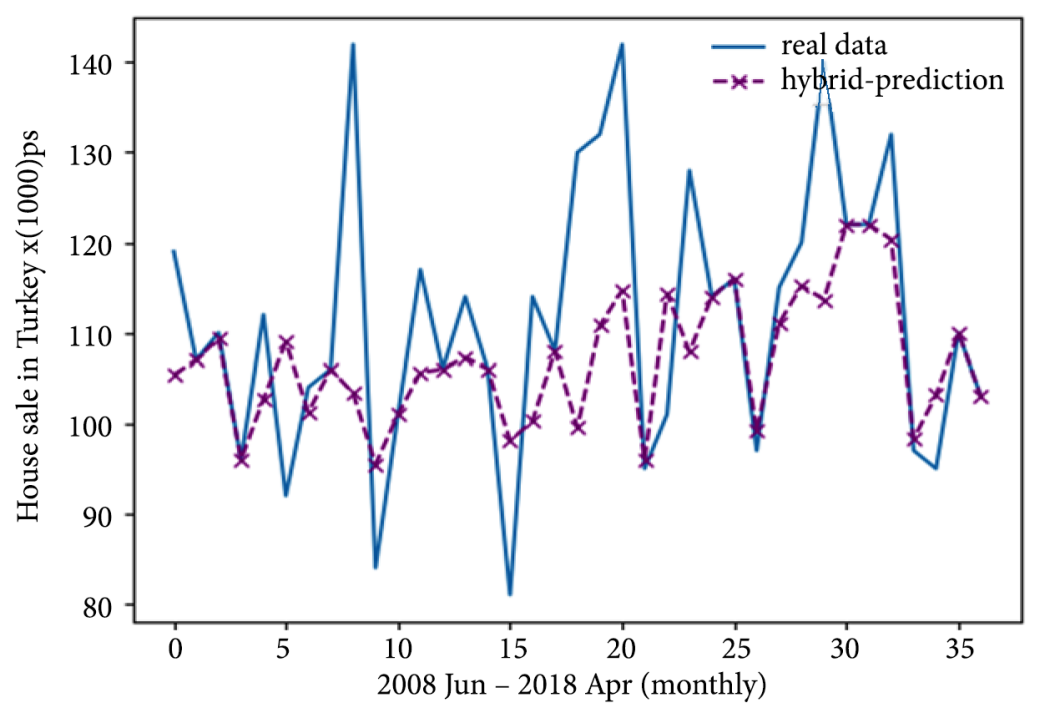

Figure 7. Hybrid model and ground truth

According to the graphical representation in Figure 7, the trend breaks of the movements are very similar. According to the success criterion evaluation, Hybrid model with LSTM 1500 epoch and ARIMA $(1,1,1)$ achieved a much lower error rate than the estimated results obtained with the individual use ARIMA and LSTM models using 0.072 MAPE value. This is an indication that the Hybrid model, as mentioned in the literature, provides better results than single models and can be used to achieve successful results.

\section{Discussion}

The housing sector has a different structure than commercial buildings, especially in countries like Turkey the consideration of "housing" as both a traditional savings tool and a necessity, strengthens the demand structure (The Association of Real Estate and Real Estate Investment Companies, 2017). The surplus in the housing supply may cause undesired price reductions, which results in firms operating in this sector to face various problems, such as not being able to sell what they produce or having to sell at a lower cost. The failure of housing supply to meet demand affects the welfare of individuals who want to buy housing as shelter or investment. Therefore, accurate estimation of real estate sales is of great importance for balancing supply and demand in the housing market.

The data used in the study are the monthly "House Sales" series from January 2008 to April 2018 taken from the electronic data distribution system of TUIK. Predictions were performed with two different models and a hybrid model obtained by combining these models. For predictions of models and comparisons of these predictions, the first 87 data of the data set were reserved for training the models and the last 37 were used as the test data. After editing the data, ARIMA was trained with LSTM and Hybrid model and the test data were estimated. All models were compared based on the estimation results. 
As seen in Table 5, the MSE values of ARIMA, LSTM and Hybrid models were calculated as 280.400, 473.346 and 175.611 respectively. Considering the MSE values, Hybrid model seems to have produced better results. MAPE values were calculated as 0.072 for Hybrid model, 0.121 for ARIMA and 0.150 for LSTM model. Similarly, in comparison with MAPE values, it was observed that the Hybrid model gave better results than the other two models.

Table 5. Comparison of all models

\begin{tabular}{|l|c|c|c|c|}
\hline \multicolumn{5}{|c|}{ Comparison of All Models } \\
\hline \multicolumn{1}{|c|}{ MODEL } & MAE & MSE & RMSE & MAPE \\
\hline ARIMA (0,1,2) & 12.801 & 280.400 & 16.745 & 0.121 \\
\hline LSTM (1000 epoch) & 17.953 & 473.346 & 21.757 & 0.150 \\
\hline HYBRID (LSTM 1500 epoch-ARIMA (1,1,1)) & 8.847 & 175.611 & 13.252 & 0.072 \\
\hline
\end{tabular}

Considering the MSE values, the hybrid model achieved a performance increase of $43 \%$ compared to the predictions made with the ARIMA model and a $49 \%$ performance increase compared to the predictions made with the LSTM model. Similarly, the hybrid model achieved $34 \%$ better predictions than the ARIMA model and $40 \%$ better than the LSTM model in terms of MAPE success criteria. Both this study and many of the other studies in the literature conclude that making predictions by combining multiple methods that can model different functional relationships in the data set instead of estimating the time series with a single method clearly produce more effective results. The findings obtained in the study also confirm this.

In the study, an estimation was made for monthly housing sales in Turkey using the methods described above. The data was not estimated by being processed in the program just once, but the process was repeated dozens of times until realistic values were obtained. As seen above, the error values for each method are quite low. This accuracy of prediction in real estate sales with regards to a more balanced supply and demand will provide guiding information about the future to the Turkish real estate sector, in which the sales forecast is carried out, and the firms in the sector. This method will also serve as a model in different countries that are confined to only one study in literature review; it will show that it's possible to carry out sales predictions and not be limited to price predictions regarding housing.

In addition, the method used is adaptable to the home sales forecast for any country or state anywhere in the world because it does not consider a specific situation in the country where the estimate is made. Many different methods can be used for estimation and it may be possible to obtain different results from each method. For this reason, more than one method was used in the study and the results obtained from each method were compared in terms of their proximity to the real values. Additionally, the estimation of housing sales with the use of the same or different methods can also made based on various criteria. This is an important indication that there is no method limitation for similar studies that can be conducted later. 


\section{Conclusions}

The main goal of this study is to provide reliable and accurate estimation studies in the housing sector by using methods with a scientific basis and to ensure that housing sales are estimated as close as possible to the real value. This will help to obtain information to support the decision-making process of shareholders in the sector. This study also demonstrated the availability of different models as a tool for predicting housing sales. The purpose of using different models and hybrids developed with those different models instead of being limited to a single model is to determine the configuration that gives the lowest mean squared square root (RMSE) and mean absolute error (MAE) values. For this purpose, ARIMA, LSTM and HYBRID model formed from these two models have been used because of their widespread use in forecasting studies in social sciences. The HYBRID model produced the best performance among these three models.

In the literature review, only one estimation study was found regarding the sales of houses. This study is an application for estimating housing sales and prices made with the use of search engines. Therefore, this study out about housing sales in Turkey is expected to make a major contribution to the literature in terms of its subject matter. We believe that this study, which examines housing sales figures of the past years and employs time series analysis, will pioneer studies that will predict the housing sales in Turkey or in different countries. In addition, with the correct estimation of housing sales; it will be possible to make predictions about the future of the country's economy and to create useful information for other sectors affected by the housing sector.

Due to restrictions of time and resources, the number sales was estimated on a country basis rather than city and as monthly data in this study. In addition, the publication of housing sales by TUIK in 2008 has limited the number of data to be used. The estimation of housing sales could be made for each city or a region of a country with classification criteria such as first or second hand sales, or sales to citizens or foreigners, instead of considering a whole country because of time restrictions in this study.

\section{References}

Adeva, J. J. G., Beresi, U. C., \& Calvo, R. A. (2005). Accuracy and diversity in ensembles of text categorisers. CLEI Electronic Journal, 8(2), 1-12. Retrieved from https://pdfs.semanticscholar.org/efb5/57 12e52ad81778706ae8ba774c7ec65eb84e.pdf

Aladağ, Ç. H., Eğrioğlu, E., \& Kadılar, C. (2009). Forecasting nonlinear time series with a hybrid methodology. Applied Mathematics Letters, 22, 1467-1470. https://doi.org/10.1016/j.aml.2009.02.006

Albayrak, A. S. (2010). ARIMA forecasting of primary energy production and consumption in Turkey: 1923-2006. Enerji, Piyasa ve Düzenleme, 1(1), 24-50. Retrieved from https://asalbayrak.files. wordpress.com/2014/10/d13.pdf

Atienza, R. (2017). LSTM by example using tensorflow (text generate). Retrieved from https://towardsdatascience.com/lstm-by-example-using-tensorflow-feb0c1968537

Babu, C. N., \& Reddy, B. E. (2014). A moving-average filter based Hybrid ARIMA-ANN model for forecasting time series data. Applied Soft Computing, 23, 27-38.

https://doi.org/10.1016/j.asoc.2014.05.028 
Choi, H. K. (2018). Stock price correlation coefficient prediction with ARIMA-LSTM hybrid model. Seoul, Korea: Korea University. Retrieved from https://arxiv.org/pdf/1808.01560v5.pdf

Contreras, J., Espinola, R., Nogales, F., \& Conejo, A. (2003). ARIMA models to predict next-day electricity prices. IEEE Transactions on Power Systems, (pp. 1014-1020). Retrieved from http://halweb. uc3m.es/esp/Personal/personas/fjnm/esp/papers/ARIMAprices.pdf

Ediger, V. Ş., \& Akar, S. (2007). ARIMA forecasting of primary energy demand by fuel in Turkey. Energy Policy, 35(3), (pp. 1701-1708). https://doi.org/10.1016/j.enpol.2006.05.009

Erdoğdu, E. (2007). Electricity demand analysis using cointegration and ARIMA modelling: a case study of Turkey. Energy Policy (pp. 1129-1146). Retrieved from https://mpra.ub.uni-muenchen. de/19099/

The Association of Real Estate and Real Estate Investment Companies (Gayrimenkul ve Gayrimenkul Yatırım Ortaklığı Derneği), 2017. Türkiye Gayrimenkul Sektörü 2017 4. Çeyrek Raporu, İstanbul: GYODER. https://www.gyoder.org.tr/yayinlar/gyoder-gosterge

Greenwood, J., \& Hercowitz, Z. (1991). The allocation of capital and the time over the business cycle. Journal of Political Economy, 99 (pp. 1188-1214). Retrieved from http://www.jeremygreenwood. net/papers/gherc91.pdf

He, G., \& Deng, Q. (2012). A Hybrid ARIMA and Neural network model to forecast particulate. Matter Concentration in Changsha. Retrieved from https://pdfs.semanticscholar.org/521f/542ebf4e11ae2d 456d9733824327da325749.pdf

Hocaoğlu, F. O., Kaysal, K., \& Kaysal, A. (2015). Hybrid model for load forecasting (ANN and Regression). Akademik Platform (pp. 33-39). Retrieved from http://dergipark.gov.tr/download/articlefile/25197

Hochreiter, S., \& Schmidhuber, J. (1997). Long sort term memory. Neural Computation (pp. 1735-1780). https://doi.org/10.1162/neco.1997.9.8.1735

Ioannou, K., Birbilis, D., \& Lefakis, P. (2011). A method for predicting the possibility of ring shake appearance on standing chestnut trees. Journal of Environmental Protection and Ecology (pp. 295-304). Retrieved from http://www.jepe-journal.info/vol-12-no-1

Kang, E. (2018). Generating text using an LSTM network. Retrieved from https://github.com/llSourcell/ LSTM_Networks/blob/master/LSTM\%20Demo.ipynb

Khashei, M., H. S. B. M. (2008). A new hybrid artificial neural networks and fuzzy regression model. Fuzzy Sets and Systems, 159, 769-786. https://doi.org/10.1016/j.fss.2007.10.011

Koutroumanidis, T., Ioannou, K., \& Arabatzis, G. (2009). Predicting fuelwood prices in Greece with the use of ARIMA models, artificial neural networks and a Hybrid ARIMA-ANN Model. Energy Policy, 37, 3627-3634. https://doi.org/10.1016/j.enpol.2009.04.024

Koutroumanidis, T., Ioannou, K., \& Zafeiriou, E. (2011). Forecasting bank stock market prices with a hybrid method: the case of Alpha bank. Journal of Business Economics and Management, 12(1), 144-163. https://doi.org/10.3846/16111699.2011.555388

Lin, T., Guo, T., \& Aberer, K. (2017). Hybrid neural networks for learning the trend in time series (pp. 2273-2279). Melbourne, Australia. Retrieved from https://dl.acm.org/citation.cfm?id=3172204

Namın, S. S., \& Namın, A. S. (2018). Forecasting economic and financial time series: ARIMA vs. LSTM. Lubbock, TX, USA: Texas Tech University. Retrieved from https://arxiv.org/ftp/arxiv/papers/1803/1803.06386.pdf

Newbold, P. (1983). ARIMA model building and the time series analysis approach to forecasting. Journal of Forecasting, 2(1), 23-35. https://doi.org/10.1002/for.3980020104

Oliveira, M., \& Torgo, L. (2014). Ensembles for time series forecasting. JMLR: Workshop and Conference Proceedings, 39, 360-370. http://ds2014.ijs.si/lbp/DS2014_LBP_Oliveira.pdf 
Opitz, D., \& Maclin, R. (1999). Popular ensemble methods: an empirical study. Journal of Artificial Intelligence Research (pp. 169-198). https://doi.org/10.1613/jair.614

Pablo, B. J., Hilda, C., Xavier, A., Diego, J. J., Felipe, S., \& Henry, B. (2016). Artificial neural network and Monte Carlo forecasting with generation of L-scenarios. Intl IEEE Conference on Ubiquitous Intelligence \& Computing (pp. 665-670) Toulouse, France.

https://doi.org/10.1109/UIC-ATC-ScalCom-CBDCom-IoP-SmartWorld.2016.0110

Papagera, A., Ioannou, K., Zaimes, G., Iakovoglou, V., \& Simeonidou, M. (2014). Simulation and prediction of water allocation using artificial neural networks and a spatially distributed hydrological model. Agris on-line Papers in Economics and Informatics, 6(4), 101-111. Retrieved from https:// ageconsearch.umn.edu/record/196580

Sagheer, A. \& Kotb, M., 2019. Time series forecasting of petroleum production using deep LSTM recurrent networks. Neurocomputing, 323(5), 203-213. https://doi.org/10.1016/j.neucom.2018.09.082

Sallehuddin, R., Shamsuddin, S. M. H., Hashim, S. Z. M., \& Abraham, A. (2007). Forecasting time series data using hybrid grey relational artificial neural network and auto regressive integrated moving average model. Neural Network World (pp. 573-605). Retrieved from http://citeseerx.ist. psu.edu/........./doi=10.1.1.218.5755\&rep=rep1\&type $=$ pdf

Sar1, M. (2016). Artificial neural networks and sales demand forecasting application in the automotive industry. Sakarya Univercity, Sakarya.

Sugiartawan, P., Pulungan, R., \& Sari, A. K. (2017). Prediction by a hybrid of wavelet transform and long-short-term-memory neural network. International Journal of Advanced Computer Science and Applications, 8(2), 326-332. https://doi.org/10.14569/IJACSA.2017.080243

Wu, L., \& Brynjolfsson, E. (2015). The future of prediction: how Google searches foreshadow housing prices and sales. In: Economic Analysis of the Digital Economy. Chicago: University of Chicago Press (pp. 89-118). https://doi.org/10.7208/chicago/9780226206981.003.0003

$\mathrm{Xu}$ et al. (2019). A hybrid modelling method for time series forecasting based on a linear regression model and deep learning. Applied Intelligence, 1-14. https://doi.org/10.1007/s10489-019-01426-3

Yu, L., Jiao, C., Xin, H., Wang, Y., \& Wang, K. (2018). Prediction on housing price based on deep learning. International Journal of Computer and Information Engineerin, 12(2), 90-99. https://doi.org/10.5281/zenodo.1315879

Zhang, G. P. (2003). Time series forecasting using a Hybrid ARIMA and neural netwok model. Neurocomputing, 50, 159-175. https://doi.org/10.1016/S0925-2312(01)00702-0 